\title{
SURGERY AT DAYBREAK OF THE NEW MILLENNIUM: TRADITIONS AND INNOVATIONS
}

In recent years, colorectal surgery faces a series of challenges associated with the interdisciplinary approach in the biomedicine and clinical medicine of the new millennium. The Department of General and Operative Surgery at the Medical University "Professor Paraskev Stoyanov" of Varna has already organized 14 national conferences of coloproctology with rising international participation. The present jubilee Fifteenth International Congress of Colorectal Surgery represents the continuation of these conferences of coloproctology. It preserves the best practices and traditions and, in the same time, it traces the pathway to new innovations and scientific advances in this field of increasing socio-medical importance worldwide. It should be emphasized that these traditional conferences have substantially contributed to mutual exchange of experience and methodology as well as of theoretical and practical achievements between scientists and practitioners. It has considerably extended the interpersonal contacts and fruitful interrelations within the medical class and with the public as well.

All these scientific forums held in Varna have united the efforts of numerous eminent researchers from Bulgaria and abroad by emphasizing the significance of the prevention, diagnosis and management of the diseases of colon and rectum. The main objective of the present International Congress of Colorectal Surgery is to further set up the achievements as well as to consider the innovations in translational and personality-oriented medicine along with the modern diagnostic and therapeutic modalities available. This Congress will undoubtedly answer the hot-topic questions in the broad field of colorectal surgery and serve as an example for fruitful collaboration between Bulgarian authors and their colleagues from other countries.

The present special issue of Scripta Scientifica Medica contains several articles dealing with a variety of interesting and actual issues in coloproctology.

P. Vladova and S. Iliev demonstrate the consensus, the modern solution and the recommendations of the European Neuroendocrine Tumor Society by 2017 concerning the surgical tactics in appendicular neurendocrine neoplasms. The authors analyze the survival following different surgical interventions. The relationship between tumour size and choice of the surgical method is clearly outlined.

Despite the contradictory opinions about sentinel lymph node labelling in colorectal cancer (CRC), V. Georgiev et al. show that this method presents with definite benefits for a more qualitative tumour staging. The authors prove that in spite of the additional difficulties during laparoscopic surgery, usage of this method facilitates surgeon's work when defining the lymph drainage under the conditions of a reduced tactile sensation.

It is noteworthy that in recent years, laparoscopic surgery rapidly advances and occupies an essential place in a variety of abdominal operations. In their paper, V. Marinov et al. investigate the indications for palliative laparoscopic large bowel resections which are widely discussed in the literature available and then share their own experience. Minimal surgical trauma, short restoration period and properly defined indications are strictly considered when planning these palliative resections in the patients with a disseminated CRC. The achieved goal consists of the reduced risk of primary tumour-related complications and warranting the good quality of life of these patients.

D. Mladenovich shares his clinical experience with anal fistula management. The author concisely describes the newest surgical techniques which are applied for the treatment of this disease. Here belong the ligation of intersphincteric fistula tract, endorectal advancement flap, video-assisted anal fistula treatment, fistula tract laser closure, fibrin sealants and anal fistula plug, and adipose-derived stem cells.

Nowadays, the question which of these methods, laparoscopic or open surgery, provides better results in low rectal cancer is subject of intensive debate. G. Kostov's team comparatively follows-up the treatment of 34 
patients after the application of the laparoscopic approach and that of 42 patients who undergo conventional surgery. The analysis of a series of parameters such as operative time, blood loss, early postoperative complications, etc. demonstrates that the laparoscopic surgery proves to be more beneficial. However, in order to establish this modern surgical approach as a gold standard in colorectal surgery, numerous additional innovations should be developed and introduced in practice.

Appendectomy represents another surgical intervention in which there are no exactly defined criteria for the choice between the laparoscopic and open surgical approach yet. In their retrospective study of a total of 798 patients with diagnosed appendicitis, A. Nikolovski et al. establish that each approach could be the method of choice and the concrete usage of one or another method should be based either on surgeon's experience, or on patient's preference.

I. Novakov reports a relatively rare localization of a metastatic CRC in the pleural cavity presenting with malignant pleurisy formation. Based on the follow-up examination of 12 patients the author draws the conclusion that in every patient, pleural metastasis has occurred after a mean interval of $1,8 \pm 0,7$ years after the radical surgical intervention. Successful pleurisy evacuation has been accomplished by means of tube thoracostomy.

S. Glinkov et al. share their experience with the surgical behaviour in three patients admitted to hospital under emergency conditions on the occasion of massive bleeding which source has not been detected by means of routine endoscopy. There is an identical operative finding in these patients consisting in the presence of multiple polyposis in the proximal intestine. In these cases, the rapid surgical intervention results in good outcome.

D. Hadzhiev et al. present the results from their retrospective investigation of 547 anorectal abscess patients. According to these authors, the surgical tactics based on the individualized and differentiated approach to such patients in specialized clinics of coloproctology ensures both effective and radical management and, at the same time, avoid a secondary abscess or a chronic fistula formation.

According to I. Lyutakov et al., the difficult localization, polyp size and presence of synchronous lesions in left and right colon call for application of hybrid resection techniques. With their case report, these authors demonstrate that the combination of modern endoscopic therapeutic methods and laparoscopic surgery enables the removal of large tubulovillous polyps and adenocarcinomas of the large bowel.

A. Karashmalakov et al. describe the treatment of enterovesical fistula in four patients. These patients undergo open surgery with the main purpose to perform an intestinal stoma thus avoiding the contamination and allowing the primary urinary bladder emptying. The authors conclude that intestinal passage interruption and primary urinary bladder restoration represent reliable approaches to enterovesical fistula management.

The articles in this special issue of Scripta Scientifica Medica deserve the attention of the international readership.

Professor Krasimir Ivanov, MD, PhD, DSc

Special issue editor

President of the Fifteenth International Congress of Colorectal Surgery 